\title{
Thermal Fault Diagnosis of Transmission System in Automatic Production Machinery and Equipment and Reliability Analysis
}

\author{
Yan Cheng \\ School of Mechatronics Engineering, Zibo Vocational Institute, Zibo 255314, China
}

Corresponding Author Email: 11304@zbvc.edu.cn

https://doi.org/10.18280/ijht.390603

Received: 3 September 2021

Accepted: 10 November 2021

\section{Keywords:}

automatic production machinery and equipment, transmission system, transmission device, thermal fault diagnosis, reliability analysis

\begin{abstract}
The thermal fault diagnosis of automatic production machinery and equipment and the analysis of its reliability are of positive meanings for promoting the advancement of diagnosis technology and guaranteeing the safe and reliable operation of the equipment. For this reason, this paper attempts to research the thermal fault diagnosis of transmission system in automatic production machinery and equipment and analyze its reliability. At first, with a transmission device as an example, thermal fault diagnosis was performed on the device and its reliability was analyzed; then, the paper gave the overall structure of the thermal fault diagnosis system and elaborated on the diagnosis flow. After that, the distribution of water temperature in radiator water channels was described in detail, and a temperature model of the transmission device were constructed. At last, this paper fully considered the possible interference factors in actual production, and used 3 kinds of parameter models for systematic identification, and the experimental results proved the effectiveness of the proposed model.
\end{abstract}

\section{INTRODUCTION}

There is a wide variety of automatic production machinery and equipment, when the devices are running, some of the components could be used cooperatively with intelligent, automatic, digital, networked controllers and sensors to perform various mechanical motions and satisfy users' personalized requirements to the greatest extent [1-7]. These automatic production machinery and equipment are generally consisted of different modules, including driver, speed change, transmission, work, braking, protection, lubrication, and cooling, etc., and they are widely used in industry, agriculture, commerce, service, and other fields [8-10].

In fault diagnosis of automatic production machinery and equipment, more than $60 \%$ of the faults are electrical malfunction [11-16]. Since the internal environment of such equipment usually has the characteristics of large current, high voltage, and slow heat dissipation, when operating continuously for a long time, the temperature rise will affect the operational stability of the equipment, and further resulting in thermal failure of the equipment [17-21]. Manual hand-held infrared thermometers can measure the temperature of the equipment easily at close range, and accurately locate the positions of the thermal faults, but this method requires large workload and it couldn't monitor the working status of the equipment in real time [22-25]. Therefore, thermal fault diagnosis and reliability analysis play a positive role in promoting the advancement of fault diagnosis technologies and ensuring safe and reliable operation of automatic production machinery and equipment, and they are of certain practical value.

Infrared thermal imaging can diagnose faults in electrical equipment without interrupting the operation. Li [26] introduced the principle of infrared imaging technology, and gave a detailed analysis and introduction of effective diagnosis methods; through actual application examples of infrared image segmentation algorithm in internal and external fault detection of electrical equipment, the effectiveness of the algorithm was verified in the paper. Aiming at the problem that the infrared image of a single band cannot fully reflect the real temperature information of the target, Lu et al. [27] explored a thermal fault diagnosis method for electrical equipment in substations based on image fusion, proposed an infrared image registration and fusion algorithm for the said matter, and constructed an image segmentation and recognition model based on mask area Convolutional Neural Networks (CNN); then, the paper elaborated on the specific steps of thermal fault diagnosis of electrical equipment in substations and demonstrated the effectiveness of the model through experiments. Wei et al. [28] proposed a thermal fault diagnosis model based on improved Bayesian optimization; in order to make use of the effective network structure of image classification, it created ResNet10 and ResNet14 with smaller parameter sizes based on $\mathrm{CNN}$ and residual blocks, selected hyper parameters using an improved Bayesian optimization method based on Gaussian process, and set constraints on validation accuracy and parameter sizes to improve the performance of the algorithm. Zheng et al. [29] established a balance equation between heat generation and heat dissipation, designed a thermal experiment, and deduced the relationship between the heating of busbar joint and operating environment according to the experimental results; the paper also proposed that, under different operating conditions and environments, the key to determining the specific quantitative relationship between temperature and various influencing factors is ascertaining some operating parameters of the operating environment. Yang et al. [30] proposed a new method for common fault diagnosis of large equipment in thermal power 
plants; based on data collected from the SCADA system, the paper developed a hybrid intelligent data mining framework to extract hidden diagnostic information, and tested it with all data in the thermal power plant SCADA system database which is used for boiler fault diagnosis.

For the purpose of effectively solving problems lying in automatic production machinery and equipment such as inability to perform real-time monitoring of thermal faults, low reliability of power supply, and delay in early warning of heating state, and finally realizing accurate diagnosis and timely early warning of thermal faults, this paper conducted related research and the main contents of this paper are arranged as follows: 1) research thermal fault diagnosis and its reliability with the transmission device of automatic production equipment as the example, and give a detailed introduction to the overall structure of the thermal fault diagnosis system and the corresponding diagnosis flow; 2) describe the distribution of water temperature in radiator water channels using coil resistance value, and build a temperature model for the transmission device of automatic production equipment; 3) fully consider the interference factors in actual production, and apply the three parameter models: the Least Square Method (LSN) parameter model, the Radial Basis Neural Network (RBNN) parameter model, and the Generalized Regression Neural Network (GBNN) parameter model, for systematic identification; then, compare the performance of the three parameter models and use experimental results to verify the effectiveness of the proposed temperature model.

\section{FLOW OF THERMAL FAULT DIAGNOSIS}

If a large-capacity automatic production equipment is running at full load or over load status, the equipment will have heat loss and generate a huge amount of heat in the enclosed environment inside the equipment, and the heat must be effectively dissipated through the cooling system, otherwise the high temperature generated by the accumulation of heat will cause huge damages to iron core, coil, lead wire, insulating material and other structural parts of the equipment, seriously endangering the safe and stable operation of the equipment. In a large-capacity automatic production equipment, the transmission device is the core component, and water-cooling heat dissipation device is usually adopted as its cooling system, therefore, this paper took the transmission device of automatic production equipment as the example to carry out thermal fault diagnosis and reliability analysis. Figure 1 shows the overall structure of the thermal fault diagnosis system. According to the general reasoning idea of thermal faults, the system can be divided into several modules, including: fault rule library, mathematical model library, operation database, artificially-aided diagnosis information preprocessing, logical reasoning engine, thermal fault determination and processing, equipment control, and humancomputer interaction interface, etc.

Combining with the actual operational characteristics of automatic production equipment, this paper gave a preliminary design of the flow of equipment thermal fault diagnosis. Figure 2 shows the specific flow of thermal fault prediction. According to the operating parameters extracted from the equipment management information system, the diagnosis system regularly judges the current working conditions of the equipment, construct standard temperature model under the premise of stable equipment operation, and set the threshold based on the standard value of each temperature measuring point. When the measured temperature value is greater than the threshold value, the diagnosis system will immediately call other operating parameters to further judge whether there is a thermal fault. Therefore, establishing the standard temperature model is very important for the thermal fault diagnosis of automatic production equipment, and it is also the primary problem to be solved in this paper.

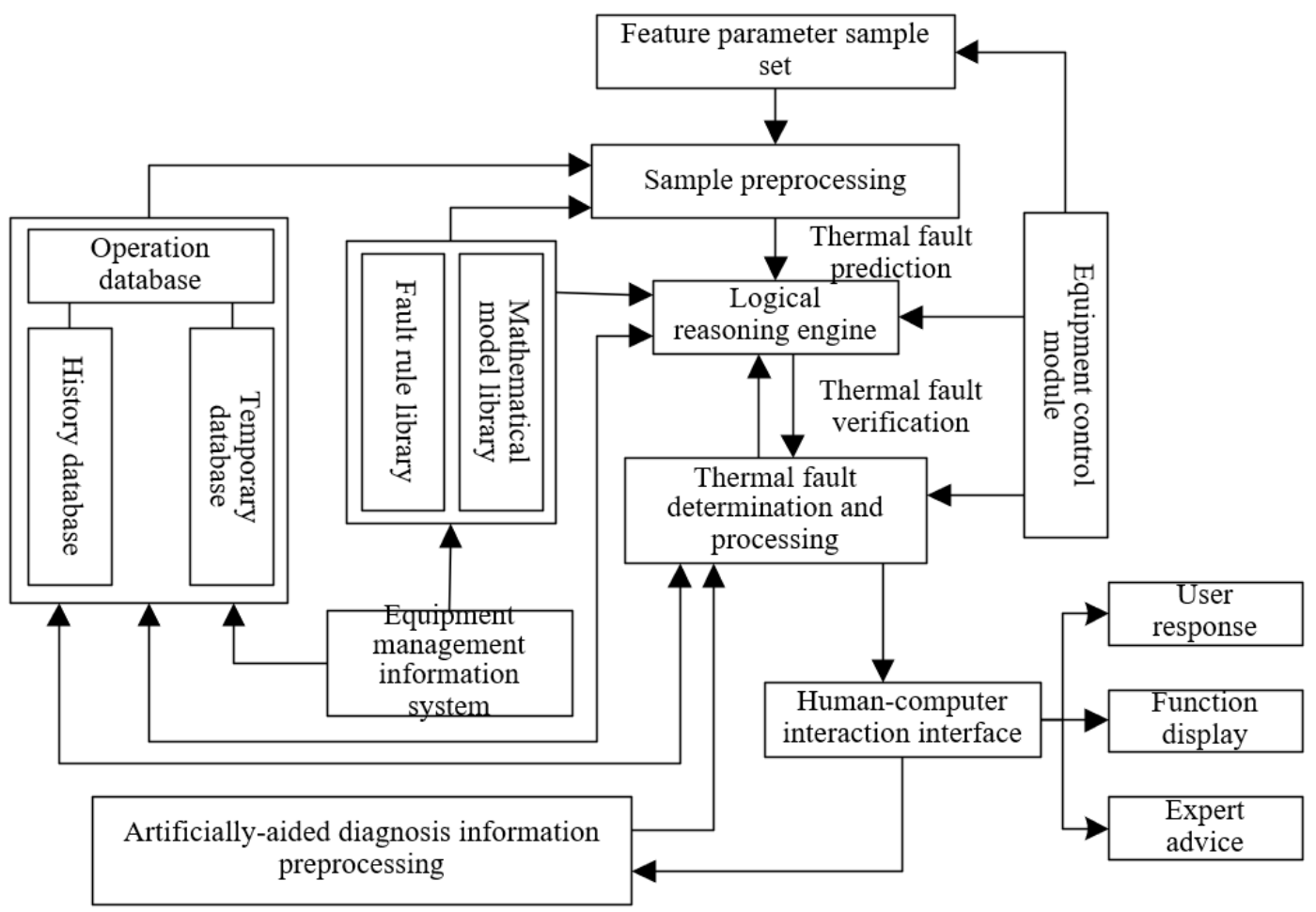

Figure 1. Overall structure of thermal fault diagnosis system 


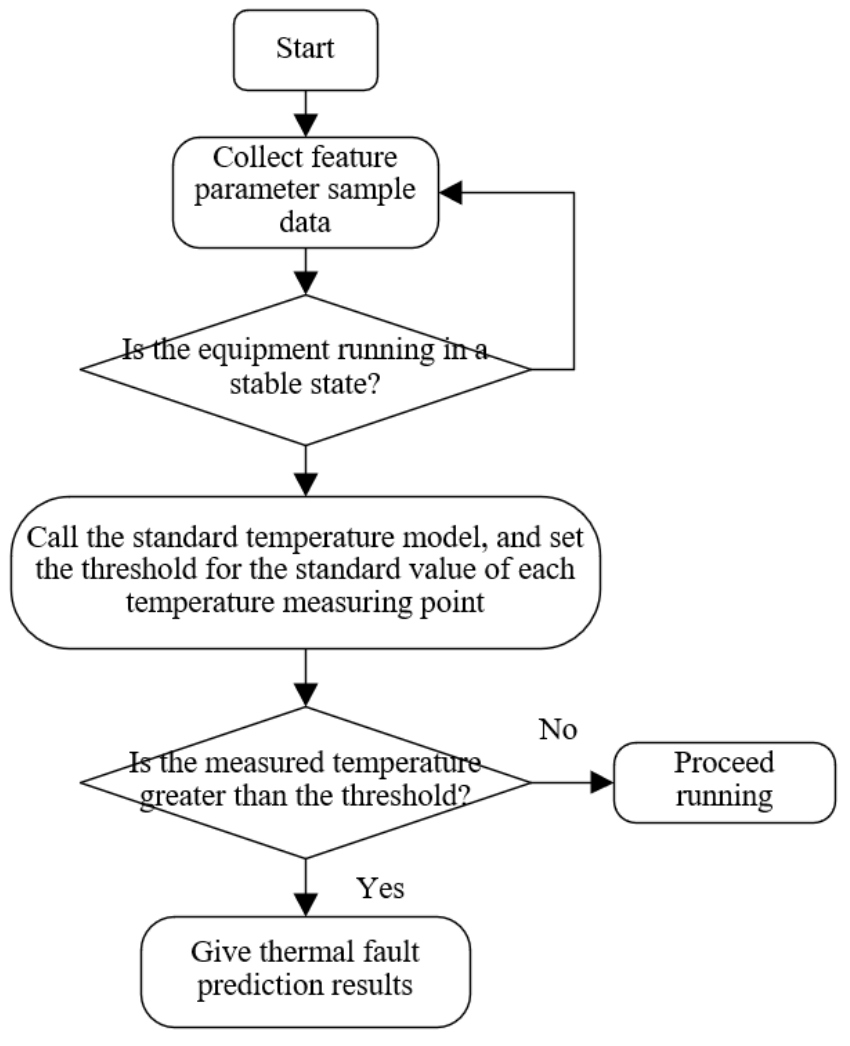

Figure 2. Flow of thermal fault prediction

\section{CONSTRUCTION OF THE TEMPERATURE MODEL}

In an automatic production equipment, between the inlet and outlet of the water cooling radiator, there're certain differences in the water temperature at different positions due to the different lengths of radiator water channels in the winding coils of the motor of the transmission device, but there's a certain relationship between the coil resistance and the water temperature in water channels, so this paper used the resistance value of the coil to characterize the distribution of water temperature in radiator water channels. Assuming: $\beta=1 /\left(235+\psi_{0}\right)$ represents the temperature coefficient, $\psi_{0}$ takes the normal temperature $15^{\circ} \mathrm{C}$, then the coil resistance is $E=\sigma_{0} C U\left[1+\beta\left(\psi-\psi_{0}\right)\right] C U S$. According to this equation, as the temperature of the cooling water rises, the resistance of the coil will increase gradually.

To this end, in this paper, an infinitesimal section with a length of $d a$ was taken from position $\tau$ of the winding coil of the transmission device, assuming: $\psi_{a \tau}(a)$ represents the average temperature of copper at $\tau, S$ represents the equivalent cross-sectional area, $\xi_{\tau}$ represents the skin effect coefficient, then Formula 1 gives the formula for calculating the equivalent resistance of the winding coil:

$$
E_{\tau}(a)=\xi_{\tau} \sigma_{0}\left[1+\beta \psi_{a \tau}(a)-\psi_{0}\right] \frac{d a}{S}
$$

Assuming: $C U_{\tau}$ represents the current in the hollow winding coil, then the heat $W_{1 \tau}$ generated by the resistance of the winding coil in the $d a$ section can be calculated by Formula 2:

$$
W_{1 \tau}=C U_{\tau}^{2} E_{\tau}(a)=C U_{\tau}^{2} \xi_{\tau} \sigma_{0}\left[1+\beta \psi_{a \tau}(a)-\psi_{0}\right] \frac{d a}{S}
$$

Assuming: $\psi_{\tau}(a)$ represents the temperature of cooling water at $\tau, d \psi_{\tau}(a)$ represents the difference of cooling water temperature of $d a$ section, $v$ represents the flow rate of water, $\mathrm{SH}$ represents the specific heat of water, then the heat $W_{2 \tau}$ absorbed by water flow passing through the $d a$ section of the winding coil in unit time can be calculated by Formula 3:

$$
W_{2 \tau}=v S H d \psi_{\tau}(a)
$$

Assuming: all the heat generated by the resistance of the winding coil is taken away by the cooling water, then there is $W_{1 \tau}=W_{2 \tau}$, namely:

$$
C U_{\tau}^{2} \xi_{\tau} \sigma_{0}\left[1+\beta \psi_{a \tau}(a)-\psi_{0}\right] \frac{d a}{S}=v S H d \psi_{\tau}(a)
$$

According to Newton's law of heat dissipation, at position $\tau$, there is a temperature difference $\psi_{d \tau}(x)-\psi_{\tau}(a)$ between the winding coil and the cooling water, the winding coil transfers heat to the cooling water, and the transferred heat amount $W_{3 \tau}$ can be calculated by Formula 5:

$$
W_{3 \tau}=\beta_{1}\left[\psi_{d \tau}(a)-\psi_{\tau}(a)\right] G I_{\tau} d a
$$

Assuming: $G I_{\tau}$ and $r_{m}$ respectively represent the perimeter and radius of the inner cavity of winding coil, $\beta_{1}=\left(0.023 E_{g} O_{e} \mu\right) / r_{m}$ represents the surface heat dissipation coefficient, then, under the condition of reaching a relatively stable thermal equilibrium with a long heating time, there is $W_{1 \tau}=W_{2 \tau}=W_{3 \tau}$. When $a$ is 0 , the inlet water temperature of the water cooling radiator is represented by $\psi_{\tau}(0)$, then the distribution of water channels along the length of the winding coil can be calculated by Formula 6:

$$
\psi_{\tau}(a)=\left(\psi_{0}-\frac{1}{\beta}\right)+\left[\psi_{\psi M}-\left(\psi_{0}-\frac{1}{\beta}\right)\right] g^{\delta z}
$$

where,

$$
\delta=\frac{\beta \beta_{1} \xi_{\tau} \sigma_{0} C U_{\tau}^{2} G I_{\tau}}{v S H\left(S \beta_{1} S H_{\tau}-\beta_{1} \xi_{\tau} \sigma_{0} C U_{\tau}^{2}\right)}
$$

If the length of coil wire is represented by $K$, then when $a=\mathrm{K}$, the water outlet temperature of the water cooling radiator can be represented by $\psi_{\tau}(\psi)$; after bringing in $a=K$, and expanding with Taylor series, we can get:

$$
\begin{aligned}
& \psi_{\tau}(K)=\left(\psi_{0}-\frac{1}{\beta}\right)+\left[\psi_{\psi M}-\left(\psi_{0}-\frac{1}{\beta}\right)\right] \\
& \left\{\begin{array}{l}
\left.1+\left(\frac{\beta_{1} \xi_{\tau} \sigma_{0} K}{v S H S}\right) C U_{\tau}^{2}+\frac{1}{2}\left[\begin{array}{l}
\left(\frac{\beta_{1} \xi_{\tau} \sigma_{0} K}{v S H S}\right)^{2} \\
+\frac{2 \beta^{2} \xi_{t}^{2} \sigma_{0}^{2} K}{v S H S^{2} G I_{1} \beta_{1}}
\end{array}\right] C U_{\tau}^{4}+\ldots\right\}
\end{array}\right.
\end{aligned}
$$

From above analysis, it is not difficult to find that, the temperature of winding coil is related to the temperature of the inlet and outlet cooling water of the water cooling radiator, the volume of cooling water, and the current flowing through the coil, and the relationship is non-linear. 
Based on the established equivalent model, the following distribution function of winding coil temperature can be established:

$$
\begin{aligned}
& \psi_{d \tau}(K)=\left(\psi_{0}-\frac{1}{\beta}\right) \\
& +\left[\psi_{e}-\left(\psi_{0}-\frac{1}{\beta}\right)\right]\left[\frac{S \beta_{1} G I_{\tau}}{\left(S \beta_{1} G I_{\tau}-\beta_{1} \xi_{\tau} \sigma_{0} C U_{\tau}^{2}\right)}\right] z^{\delta}
\end{aligned}
$$

where,

$$
\delta=\frac{\beta \beta_{1} \xi_{\tau} \sigma_{0} C U_{\tau}^{2} G I_{\tau}}{v S H\left(S \beta_{1} G I_{\tau}-\beta_{1} \xi_{\tau} \sigma_{0} C U_{\tau}^{2}\right)}
$$

Performing power series expansion on the above formula, it's shown that, for the distribution function, except for some specific coefficients, the rest nonlinear relationships are similar to that of Formula 8.

\section{PARAMETER IDENTIFICATION OF THE TEMPERATURE MODEL}

Since some parameters of the standard temperature model of winding coil are difficult to determine, and the physical heat transfer mechanism can hardly be explored, this paper built a temperature model equivalent to the system of the automatic production equipment based on input and output data of the equipment information management system, and fully considered the possible interference factors in actual production. Further, a LSM model, a RBNN model, and a GRNN model were applied for systematic identification, and their performance was compared.

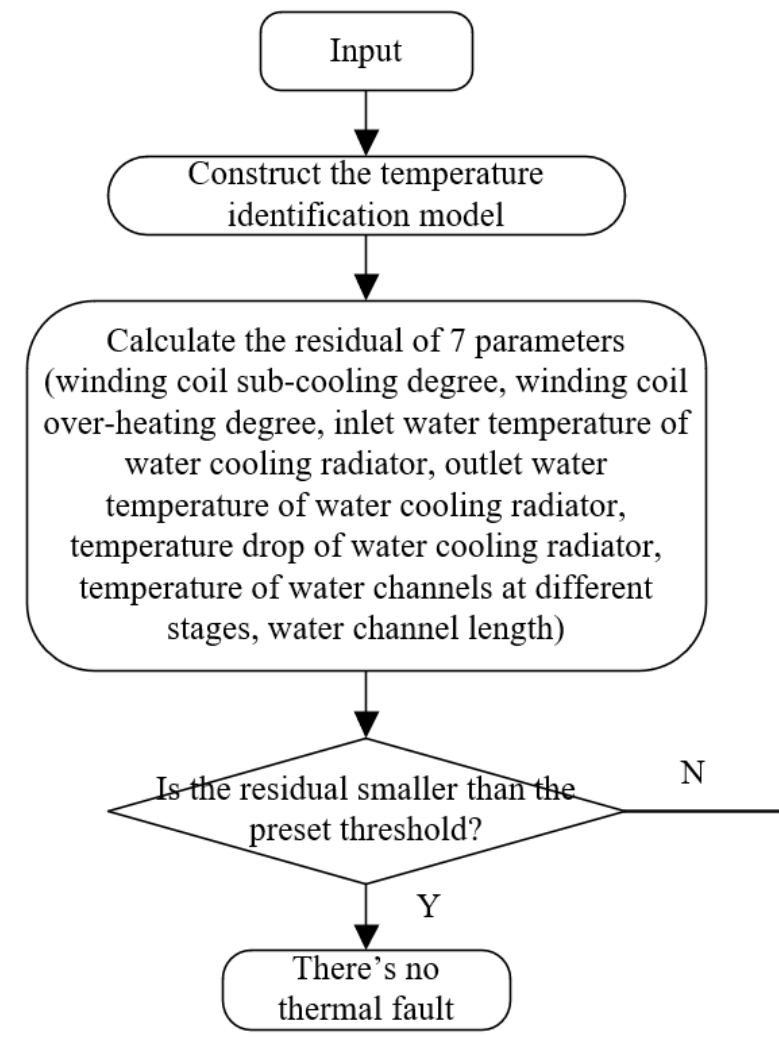

In LSM, $b$ is a known function about independent variable $A$ and undetermined parameter $Y$, which is represented by $b=g(A, Y)$. That is, $A_{i}\left(a_{1 l}, a_{2 l}, \ldots a_{o l}\right)(l=1,2, \ldots m), \quad Y=\left(y_{1}, y_{2}, \ldots y_{n}\right)$. Given $m(A, b):\left(A_{i}, b_{i}\right)(l=1,2, \ldots m)$, we can obtain a parameter $Y$ that ensures $W$ is the minimum:

$$
W=\sum_{i=1}^{m}\left[b_{i}-g\left(A_{i}, Y\right)\right]^{2}
$$

Since $b$ and $y$ have a linear relationship, this paper used a linear combination $\varphi_{1}(A), \varphi_{2}(A), \ldots \varphi_{n}(A)$ of $n(n<m)$ functions to replace the functional relationship of $m$ pairs, that is:

$$
b=y_{0}+y_{1} \phi_{1}(A)+y_{2} \phi_{2}(A)+\ldots+y_{n} \phi_{n}(A)
$$

where, $\varphi_{i}(A)(i=1,2, \ldots, n)$ is a known function of $A=\left(a_{1}, a_{2}, \ldots, a_{i}\right)$. That is, set a $M \times N$-dimensional matrix, $X=\left[P_{1}, P_{2}, \ldots P_{n}\right]$, wherein the column vectors $P_{\tau}=\left[P_{\tau}\left(A_{1}\right), P_{\tau}\left(A_{2}\right), \ldots P_{\tau}\left(A_{m}\right)\right]^{T}$ and $B=\left(b_{1}, b_{2}, \ldots b_{m}\right)^{T}$, then the equation $X^{T} X Y=X^{T} B$ is satisfied, and the following equations can be obtained:

$$
\begin{aligned}
& Y=\left(X^{T} X\right)^{-1} X B \\
& W=B^{T}(B-X Y)
\end{aligned}
$$

According to above formulas, for nonlinear parameter systems, the Newton's method can be used to linearize them at first, and then the above analysis can be used for parameter identification. In the proposed temperature model, this paper selected the standard temperature value of the motor used in the transmission system when it leaves the factory and the actual operating data for parameter identification.

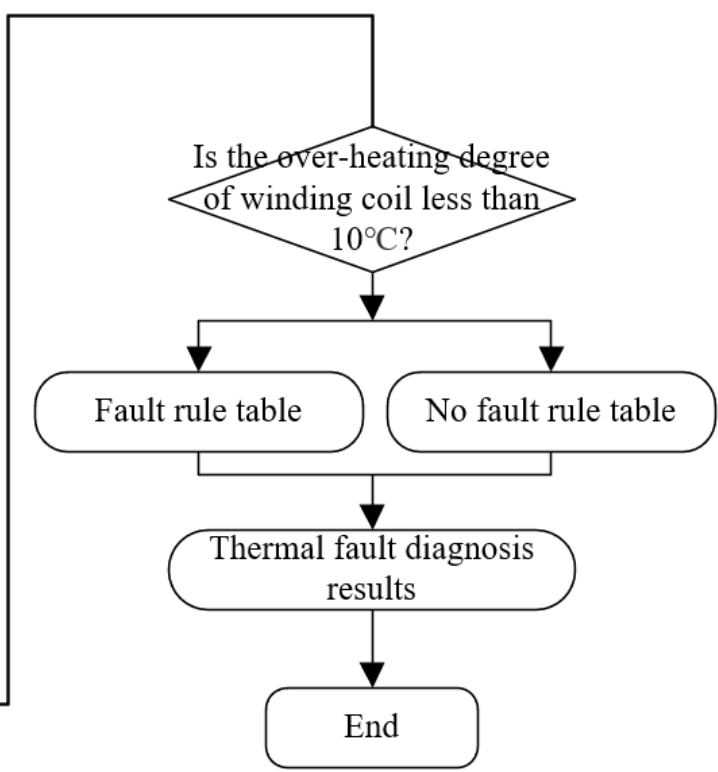

Figure 3. Flow of thermal fault diagnosis based on neural network 


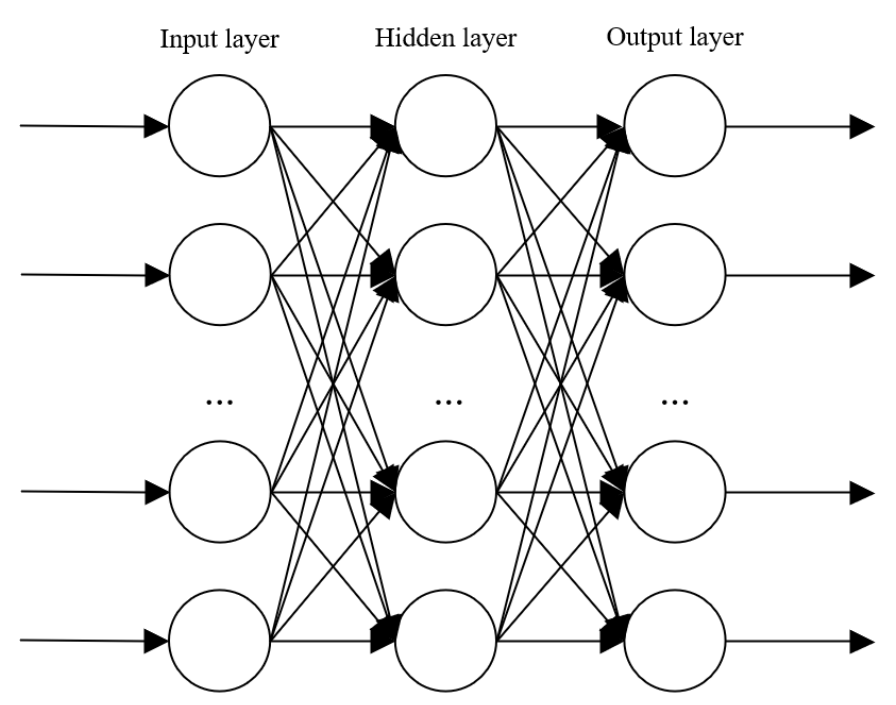

Figure 4. Structure of the RBNN model

To use RBNN and GRNN to analyze the fault characteristics of the transmission system of automatic production equipment under thermal fault state, this paper determined following feature parameters of the equipment under thermal fault state: sub-cooling degree of the winding coil, over-heating degree of the winding coil, inlet water temperature of water cooling radiator, outlet water temperature of water cooling radiator, temperature drop of water cooling radiator, temperature of water channels at different stages, and water channel length. At the same time, three parameters of outdoor dry bulb temperature, indoor dry bulb temperature and indoor dew point temperature were selected. Figure 3 shows the flow of thermal fault diagnosis based on neural network.

Figure 4 shows the structure of the RBNN model. The RBNN constructed in this paper is consisted of three layers: input layer, hidden layer, and output layer. The parameters to be predicted determined the number of neuron nodes in the output layer of the network. Assuming: $a$ represents the input sample, $b$ represents the output, $D_{L}$ represents the center of the Gaussian function, $\left\|a-D_{L}\right\|$ represents the Euclidean norm, $\rho$ represents the variance of the Gaussian function, $\theta_{i L}$ represents the weight of connection from hidden layer to output layer, $M$ represents the number of hidden layer nodes, then the function of the output layer of the network is given by the following formula:

$$
\begin{gathered}
\varphi_{L}(a)=\exp \left(-\frac{\left\|a-D_{L}\right\|^{2}}{2 \rho_{L}^{2}}\right) \\
b=g_{i}(a)=\sum_{L=1}^{M} \theta_{i L} \varphi_{L}\left(\left\|a-D_{L}\right\|^{2}\right)
\end{gathered}
$$

The specific calculation steps of the RBNN model are described in detail as follows:

Step 1: Select a set of initial center values $D_{L}$ from the input samples;

Step 2: Calculate the variance value based on Formula 17:

$$
\rho=r_{\max } / L
$$

Step 3: Calculate $b_{i}^{\prime}(m)$ based on the following formula:

$$
b_{i}^{\prime}(m)=\sum_{L=1}^{N} \theta_{l} \varphi\left[a(m), D_{l}, \rho_{l}\right]
$$

Step 4: Update the parameters of the constructed neural network as follows:

$$
\begin{gathered}
\theta(m+1)=\theta(m)+\lambda_{q} z(m) \Phi(m) \\
D_{l}(m+1)=D_{l}(m)+\lambda_{d} \frac{z(m) \theta_{l}(m)}{\rho_{l}^{2}(m)} \\
\Phi\left[a(m), D_{l}(m), \rho_{l}\right]\left[a(m)-D_{l}(m)\right] \\
\rho_{l}(m+1)=\rho_{l}(m)+\lambda_{\rho} \frac{z(m) \theta_{l}(m)}{\rho_{l}^{2}(m)} \\
\Phi\left[a(m), D_{l}(m), \rho_{l}\right]\left|\left[a(m)-D_{l}(m)\right]\right|^{2}
\end{gathered}
$$

Assuming: $b_{c}(m)$ represents the expected output of the network, $\lambda_{M}, \lambda_{d}$, and $\lambda_{\rho}$ respectively represent the learning step size of the three parameters, then there is:

$$
\begin{gathered}
\Phi(m)=\left\{\begin{array}{l}
\Phi\left[a(m) \cdot D_{1}(m), \rho_{1}\right], \\
\Phi\left[a(m) \cdot D_{2}(m), \rho_{2}\right], \\
\ldots \Phi\left[a(m) \cdot D_{m}(m), \rho_{m}\right]
\end{array}\right\}^{T} \\
z(m)=b_{i}^{\prime}(m)-b_{c}(m)
\end{gathered}
$$

Step 5: If the network converges or the termination condition is met, the iterative calculation stops, and the $b_{i}^{\prime}(m)$ value is output, otherwise, returns to Step 4.

The GRNN established based on the theory of nonlinear regression is consisted of four layers: input layer, pattern layer, summation layer, and output layer. The specific calculation steps of the model are:

Step 1: Directly pass the feature parameter input samples of the input layer to the pattern layer.

Step 2: Assume $A$ represents the test data; $A_{i}$ represents the training data corresponding to the $i$-th neuron, and $\chi$ represents the smoothing factor, then Formula 24 gives the expression of the neuron transfer function of the pattern layer:

$$
R U_{i}=\exp \left[-\left(A-A_{i}\right)^{T}\left(A-A_{i}\right) / 2 \chi^{2}\right], i=1,2, \ldots, m
$$

Step 3: Assume $v_{\tau}$ represents the weight factor of the observed value of samples, then Formulas 25 and 26 give the neuron summation formulas:

$$
\begin{gathered}
V_{Q H}=\sum_{i=1}^{m} \exp \left[-\left(A-A_{i}\right)^{T}\left(A-A_{i}\right) / 2 \chi^{2}\right] \\
V_{M j}=\sum_{i=1}^{m} v_{i} \exp \left[-\left(A-A_{i}\right)^{T}\left(A-A_{i}\right) / 2 \chi^{2}\right]
\end{gathered}
$$

Step 4: Divide $V_{M j}$ by $V_{Q H}$ to get the network output $b_{i}$, that is:

$$
b_{i}=V_{M j} / V_{Q H} j=1,2, \ldots, n
$$




\section{EXPERIMENTAL RESULTS AND ANALYSIS}

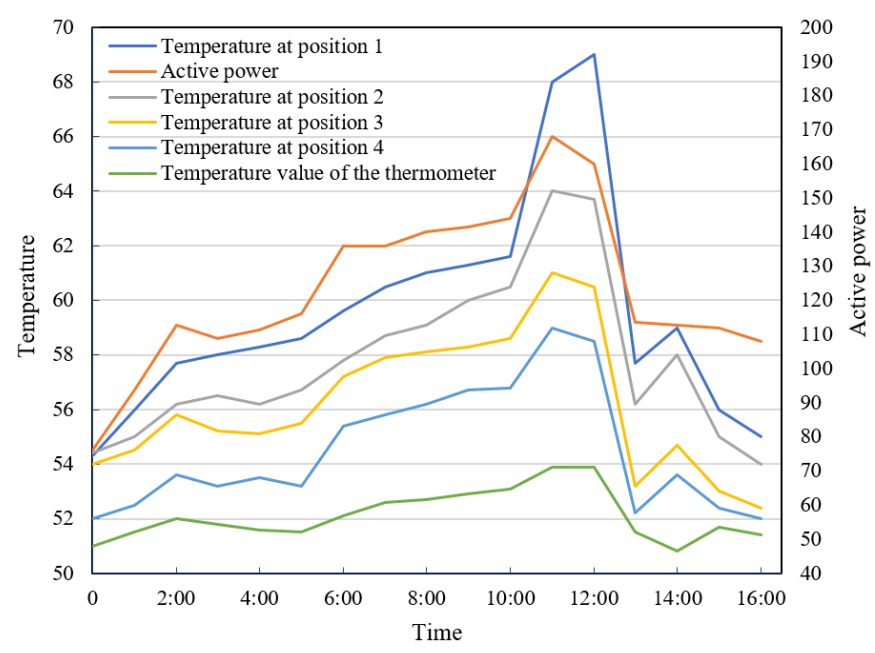

Figure 5. Temperature measured by thermometer at different positions of the transmission device

Subject in this paper is a transmission unit of an automatic coal mining device used by a coal mine in 2018. Figure 5 shows the curve of temperature measured by a thermometer at different positions of the transmission unit. According to the figure, when the coal mining load of the unit increases, in the thermal fault diagnosis system of traditional device motor, the color of the coil of 4 winding slots will turn red, and alarm content of thermal fault will be prompted. By comparing with the temperature of other winding slots, it's found that the temperature difference was relatively large, wherein the temperature difference between the slot at position 1 and the highest temperature of other normal temperature slots was $15^{\circ} \mathrm{C}$, therefore, the thermal fault alarm of the target transmission device motor is not optimistic, it needs to stop loading, and restart the unit and apply the load after the reason of thermal fault has been identified.

In this paper, 8 groups of sample data of the transmission unit of the automatic mining devices of identical model running at normal state were collected and taken as the training data of the constructed neural network models. The input of the models was 8 sets of parameter sample data, namely the outdoor dry bulb temperature, indoor dry bulb temperature, and indoor dew point temperature. And the output of the models was 7 feature parameters, namely winding coil subcooling degree, winding coil over-heating degree, inlet water temperature of water cooling radiator, outlet water temperature of water cooling radiator, temperature drop of water cooling radiator, temperature of water channels at different stages, water channel length.

Since this paper requires neural network model with output results to be as close as possible to the actually measured values, the difference between preliminarily predicted values and the experimental values was calculated, and the residual was obtained and taken as the convergence condition of the models, that is, if the residual is less than 0.001, then it's judged that the network model converges; if the preset condition is not met, the parameters of the constructed network are updated until the residual is less than the pre-set value. At this time, the output of the network model is the predicted values of feature parameters under the condition that the transmission unit is in normal operating state.

Table 1 lists the residual values of 7 feature parameters one by one. As can be seen from the table, the residual values were all less than $0.001^{\circ} \mathrm{C}$, while the instrument error of actual temperature measurement systems is usually around $0.1^{\circ} \mathrm{C}$. The maximum residual variance of the model was 0.0007 , the value was relatively small. Judging from the above data values, the RBNN model constructed in this paper is of high reliability.

Table 2 lists the calculation results of residual of different parameter models. Through comparison, it can be seen that, the residual values of RBNN were all less than 0.001 , the residual values of GRNN were all less than 0.0074 , and the maximum residual of the LSM model was 4.2614. The results showed that, the RBNN model can meet the prediction requirements, while the other two models failed, so this paper chose the RBNN model for the parameter identification of the temperature model.

Figure 6 shows the diagnosis correct rates of different-type thermal faults of transmission device under different temperature thresholds. Specifically, the fault types include: overload thermal faults, winding coil short-circuit thermal faults, frequent startup thermal faults, moisture or watercaused thermal faults, insulation and aging thermal faults, thermal fault caused by bearing lubricant shortage, and radiator failure thermal faults. According to the figure, with the increase of threshold, the correct rates of different-type thermal faults decrease gradually, and this is more obvious with overload thermal faults, frequent startup thermal faults, and radiator failure thermal faults. Judging from the corrects rate of thermal fault diagnosis of transmission device, taking $1^{\circ} \mathrm{C}$ as the temperature threshold is more appropriate.

Table 1. Residual values of RBNN model

\begin{tabular}{ccccccccc}
\hline Wording condition No. & $\mathbf{1}$ & $\mathbf{2}$ & $\mathbf{3}$ & $\mathbf{4}$ & $\mathbf{5}$ & $\mathbf{6}$ & $\mathbf{7}$ & $\mathbf{8}$ \\
\hline Feature parameter 1 & $1 \times 10^{-4}$ & $1 \times 10^{-5}$ & $2 \times 10^{-4}$ & $2 \times 10^{-4}$ & $1 \times 10^{-4}$ & $4 \times 10^{-4}$ & $1 \times 10^{-4}$ & $5 \times 10^{-4}$ \\
Feature parameter 2 & $1 \times 10^{-5}$ & $1 \times 10^{-4}$ & $2 \times 10^{-4}$ & $2 \times 10^{-4}$ & $1 \times 10^{-4}$ & $6 \times 10^{-4}$ & $1 \times 10^{-4}$ & $3 \times 10^{-4}$ \\
Feature parameter 3 & $4 \times 10^{-4}$ & $5 \times 10^{-5}$ & $2 \times 10^{-4}$ & $3 \times 10^{-4}$ & $5 \times 10^{-4}$ & $4 \times 10^{-4}$ & $4 \times 10^{-4}$ & $5 \times 10^{-5}$ \\
Feature parameter 4 & $4 \times 10^{-4}$ & $1 \times 10^{-4}$ & $2 \times 10^{-4}$ & $2 \times 10^{-4}$ & $1 \times 10^{-4}$ & $4 \times 10^{-4}$ & $1 \times 10^{-4}$ & $2 \times 10^{-4}$ \\
Feature parameter 5 & $1 \times 10^{-4}$ & $1 \times 10^{-5}$ & $2 \times 10^{-4}$ & $2 \times 10^{-4}$ & $1 \times 10^{-4}$ & $2 \times 10^{-4}$ & $5 \times 10^{-4}$ & $4 \times 10^{-4}$ \\
Feature parameter 6 & $2 \times 10^{-4}$ & $4 \times 10^{-5}$ & $3 \times 10^{-4}$ & $2 \times 10^{-4}$ & $5 \times 10^{-4}$ & $5 \times 10^{-4}$ & $1 \times 10^{-4}$ & $5 \times 10^{-4}$ \\
Feature parameter 7 & $3 \times 10^{-4}$ & $2 \times 10^{-5}$ & $2 \times 10^{-4}$ & $4 \times 10^{-4}$ & $1 \times 10^{-4}$ & $4 \times 10^{-4}$ & $7 \times 10^{-4}$ & $3 \times 10^{-4}$ \\
\hline
\end{tabular}

Table 2. Residual values of different parameter models

\begin{tabular}{cccccccc}
\hline No. of feature parameter & $\mathbf{1}$ & $\mathbf{2}$ & $\mathbf{3}$ & $\mathbf{4}$ & $\mathbf{5}$ & $\mathbf{6}$ & $\mathbf{7}$ \\
\hline RBNN & $8.1 \times 10^{-4}$ & $8.7 \times 10^{-4}$ & $5.8 \times 10^{-4}$ & $5.4 \times 10^{-4}$ & $1.6 \times 10^{-4}$ & $1.4 \times 10^{-4}$ & $1.1 \times 10^{-4}$ \\
GRNN & $1.3 \times 10^{-4}$ & $4.3 \times 10^{-3}$ & $7.2 \times 10^{-3}$ & $7.4 \times 10^{-3}$ & $4.5 \times 10^{-3}$ & $2.3 \times 10^{-4}$ & $2.2 \times 10^{-3}$ \\
LSM & 0.0148 & 0.0259 & 0.0347 & 0.0195 & 1.0274 & 0.1364 & 4.2614 \\
\hline
\end{tabular}




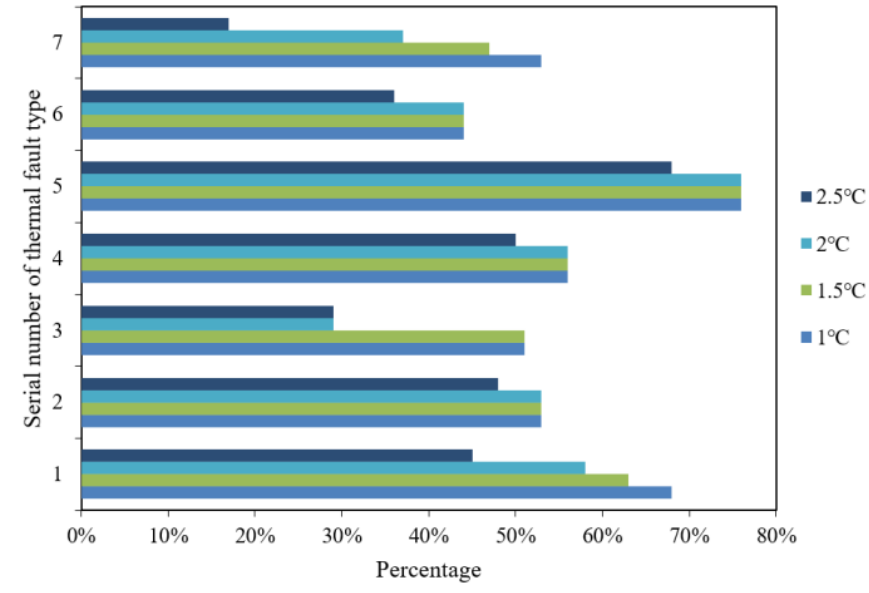

Figure 6. Correct rate of thermal fault diagnosis under different thresholds

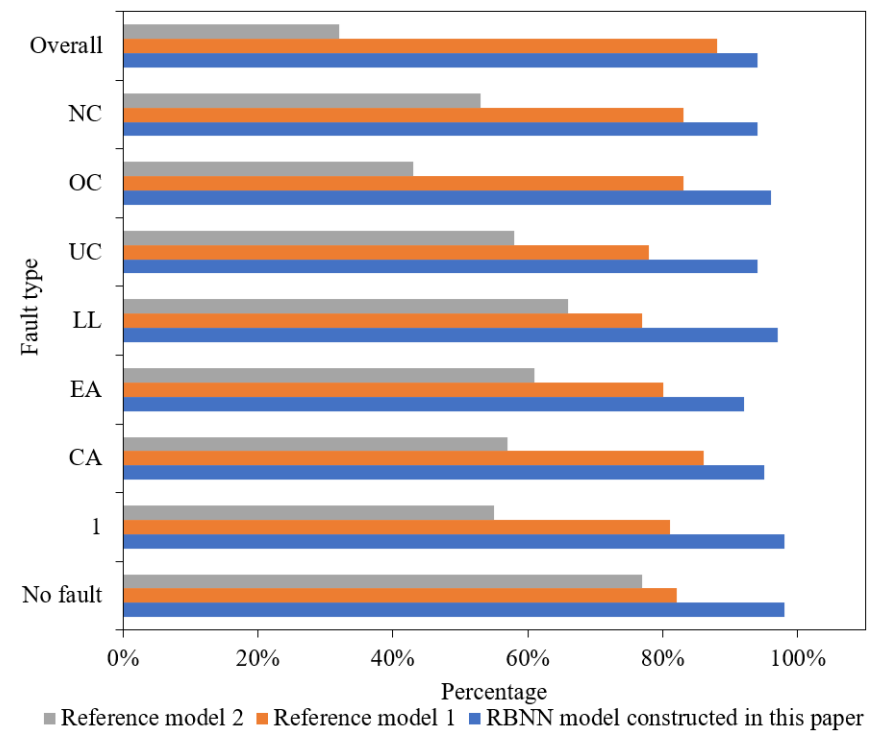

Figure 7. Correct rate of thermal fault diagnosis of different models

This paper compared and analyzed the correct rate of thermal fault diagnosis of three models: the RBNN model constructed in this paper, and two references models, the LSM model, and the GRNN model. For LSM model, the thermal fault judgment method of dividing into no fault, minor fault, and major fault was adopted; while for the RBNN model and the GRNN model, the thermal fault judgment method of taking a temperature threshold of $1^{\circ} \mathrm{C}$ was adopted.

Figure 7 is a summary of the correct rates under different thermal fault judgment methods. RBNN had the highest correct rate and the best diagnosis effect, followed by GRNN, and the correct rate of the LSM model was the lowest.

\section{CONCLUSION}

This paper studied the thermal fault diagnosis of transmission device of automatic production equipment and analyzed the reliability. Taking the transmission device of automatic production equipment as the example, this paper carried out thermal fault diagnosis and reliability analysis, and gave the overall structure of the thermal fault diagnosis system and the corresponding diagnosis flow. This paper used coil resistance to describe the distribution of water temperature in radiator water channels, and constructed the temperature model for transmission device of automatic production equipment. The interference factors in actual production were fully considered, and the system identification was carried out based on three parameter models.

In later parts, the paper plotted temperature curves of different measuring points on the transmission device of an automatic coal mining device, and found out that the thermal fault alarm state of the target transmission device motor was not optimistic. Then, for the target subject, the temperature identification model was tested, and the residual values of different parameter models were obtained. After that, the RBNN model was selected for the parameter identification of the temperature model, and the correct rates of different models under different thresholds were compared; finally, it's verified that the RBNN model had the highest correct rate.

\section{REFERENCES}

[1] Cesar, E.L., Fernandes, G.S., Kagami, M.T., Calisto, T.N. (2020). Technological obsolescence management: monitoring electrical equipment and automation systems. IEEE Industry Applications Magazine, 26(4): 82-87. https://doi.org/10.1109/MIAS.2020.2981104

[2] Yin, Y., Wang, T., Dou, L., Zhang, S., Xia, M., Xie, C. (2019, March). Standardized northbound interface testing automation on the open and disaggregated optical transport equipment. 2019 Optical Fiber Communications Conference and Exhibition (OFC), San Diego, CA, USA, pp. 1-3.

[3] Yang, Y., Fu, Y. (2021). Research on communication module of communication or automation equipment. Journal of Physics: Conference Series, 1750(1): 012075. https://doi.org/10.1088/1742-6596/1750/1/012075

[4] Rahm, J., Theißen, M., Klose, A., Soemers, M., Temmen, H., Schäfer, C., Urbas, L. (2021). Efficient automation engineering of modular process equipment assemblies using the digital twin. Chemie Ingenieur Technik, 93(12): 2081-2091. https://doi.org/10.1002/cite.202100100

[5] Azar, E.R., Kamat, V.R. (2017). Earthmoving equipment automation: A review of technical advances and future outlook. Journal of Information Technology in Construction (ITcon), 22(13): 247-265.

[6] Yamazaki, Y., Shigematsu, K., Kato, S., Kojima, F., Onari, H., Takata, S. (2017). Design method of material handling systems for lean automation-Integrating equipment for reducing wasted waiting time. CIRP Annals, 66(1): 449-452. https://doi.org/10.1016/j.cirp.2017.04.011

[7] Cui, L., Zhang, R., Liu, T., Zhang, H., Qian, C. (2017). Reliability calculation and equipment layout planning for complex distribution automation system. Automation of Electric Power Systems, 41(20): 84-91. http://dx.chinadoi.cn/10.7500/AEPS20170302005

[8] Li, D., Chen, Z., Wang, G., Zhou, X., Ren, M. (2021). The research on operation automation model for information equipment. Journal of Physics: Conference Series, 2095(1): 012048. https://doi.org/10.1088/17426596/2095/1/012048

[9] Zhu, Q., Sun, Y. (2021). Research on modeling of equipment supporting command automation system based on colored petri net. In Journal of Physics: 
Conference $\quad$ Series, $1941(1)$ : 012076 . https://doi.org/10.1088/1742-6596/1941/1/012076

[10] Ishimoto, K., Hara, S., Asakawa, A., Arai, N., Mori, R. (2019). Lightning current distribution in automation system equipment for distribution line due to direct lightning stroke. IEEJ Transactions on Power and Energy, 139(5):

372-379. https://ui.adsabs.harvard.edu/link_gateway/2019IJTPE. 139..372I/doi:10.1541/ieejpes.139.372

[11] Han, Z., Tian, L., Cheng, L. (2021). A deducing-based reliability optimization for electrical equipment with constant failure rate components duration their mission profile. Reliability Engineering \& System Safety, 212: 107575. https://doi.org/10.1016/j.ress.2021.107575

[12] He, S., Zhang, Y., Zhu, R., Tian, W. (2020). Electric signature detection and analysis for power equipment failure monitoring in smart grid. IEEE Transactions on Industrial Informatics, 17(6): 3739-3750. https://doi.org/10.1109/TII.2020.3017080

[13] Bardyk, Y.I. (2014). Modelling and assessment of chances of failure of power systems electrical equipment taking into account the after repair resource restoration level. Natsional'nyi Hirnychyi Universytet. Naukovyi Visnyk, (3): 82-90.

[14] Liu, J., Pan, X. (2017). Research on failure diagnosis model of key equipment in the electric traction system of a bullet train. Chemical Engineering Transactions, 62: 697-702. https://doi.org/10.3303/CET1762117

[15] Qiu, J., Wang, H., Lin, D., He, B., Zhao, W., Xu, W. (2015). Nonparametric regression-based failure rate model for electric power equipment using lifecycle data. IEEE Transactions on Smart Grid, 6(2): 955-964. https://doi.org/10.1109/TSG.2015.2388784

[16] Pan, L., Zhang, Y., Yu, G., Du, C. (2010). Prediction of electrical equipment failure rate for condition-based maintenance decision-making. Electric Power Automation Equipment, 2: 91-94.

[17] Yong, Y.C. (2021). Research on maintenance method of typical aircraft electronic equipment based on thermal analysis technology. 2021 IEEE International Conference on Artificial Intelligence and Industrial Design (AIID), Guangzhou, China, pp. 454-458. https://doi.org/10.1109/AIID51893.2021.9456508

[18] Qian, H., Kai, W., Hongde, X., Li, Z., Penghui, Y. (2021). Investigation on foaming and secondary reactions with a novel visual equipment and impacts on thermal analysis. Thermochimica Acta, 703: 179014. https://doi.org/10.1016/j.tca.2021.179014

[19] Siddiqui, M.N., Antonakou, E.V., Redhwi, H.H., Achilias, D.S. (2019). Kinetic analysis of thermal and catalytic degradation of polymers found in waste electric and electronic equipment. Thermochimica Acta, 675: 6976. https://doi.org/10.1016/j.tca.2019.03.001

[20] Bottaro, M., de Carvalho, T.O., Caires, L.E., Sueta, H.E., Obase, P.F., Tatizawa, H., Raposo, I.B. (2018). Analysis of asymmetrical component influence on arc current in the determination of arc thermal performance value of protective personal equipment. IEEE Transactions on Industry Applications, 55(2): 2130-2137. https://doi.org/10.1109/TIA.2018.2875852
[21] Tabadar, Z., Ansarifar, G.R., Pirouzmand, A. (2019). Thermal-hydraulic modeling for deterministic safety analysis of portable equipment application in the VVER1000 nuclear reactor during loss of ultimate heat sink accident using RELAP5/MOD3. 2 code. Annals of Nuclear Energy, $\quad$ 127: 53-67. https://doi.org/10.1016/j.anucene.2018.11.046

[22] Shen, X., Yu, X., Wang, Y., Cheng, L., Wang, D., Chen, J. (2021). three-dimensional visualization scheme of infrared thermal temperature measurement data for substaition electric power equipment. High Voltage Engineering, $\quad 47(2): \quad 387-395$. http://dx.chinadoi.cn/10.13336/j.10036520.hve.20200335

[23] Liu, K., Tang, J. K., Wang, Z., Guo, J.B., Yang, G.Y., Liang, N., Zou, Y.S. (2019). The application of a novel infrared temperature measurement system in HVDC converter valve equipment connector overheat failure prevention. Procedia Computer Science, 154: 267-273. https://doi.org/10.1016/j.procs.2019.06.040

[24] Liu, Z., Shang, Y., Su, X., Wen, J., Lin, B. (2020). Research on high temperature protection technology of infrared temperature measuring equipment in space environment. IOP Conference Series: Earth and Environmental Science, 512(1): 012167. https://doi.org/10.1088/1755-1315/512/1/012167

[25] Xu, R., Wang, L., Yin, W., Zhou, X. (2015, February). Equipment for measuring of transmission of infrared optic materials in high temperature. The International Conference on Photonics and Optical Engineering (icPOE 2014), 9449: 944936. https://doi.org/10.1117/12.2081349

[26] Li, H. (2021). Thermal fault detection and diagnosis of electrical equipment based on the infrared image segmentation algorithm. Advances in Multimedia, 2021: 9295771. https://doi.org/10.1155/2021/9295771

[27] Lu, M., Liu, H., Yuan, X. (2021). Thermal fault diagnosis of electrical equipment in substations based on image fusion. Traitement du Signal, 38(4): 1095-1102. https://doi.org/10.18280/ts.380420

[28] Wei, C., Tao, F., Lin, Y., Liang, X., Wang, Y., Li, H., Fang, J. (2019). Substation equipment thermal fault diagnosis model based on Resnet and improved Bayesian optimization. 2019 9th International Conference on Power and Energy Systems (ICPES), Perth, WA, Australia, pp. 1-5. https://doi.org/10.1109/ICPES47639.2019.9105594

[29] Zheng, Z., Song, R., Zhou, Y., Ren, C., Wang, J., Ru, Y. (2018). Study on thermal overheating faults of electrical equipment connector based on thermal balance theory. 2018 China International Conference on Electricity Distribution (CICED), Tianjin, China, pp. 555-560. https://doi.org/10.1109/CICED.2018.8592365

[30] Yang, P., Liu, S.S., Zhang, H. (2004). Fault diagnosis for large-scale equipments in thermal power plant by data mining. Control Theory \& Applications China), 21(6): 927-931. http://dx.chinadoi.cn/10.3969/j.issn.10008152.2004.06.022 